\title{
SANDWICH-TYPE THEOREMS FOR A CLASS OF INTEGRAL OPERATORS ASSOCIATED WITH MEROMORPHIC FUNCTIONS
}

\author{
NAK EUN CHO
}

\begin{abstract}
The purpose of the present paper is to investigate some subordination and superordination preserving properties of certain integral operators defined on the space of meromorphic functions in the punctured open unit disk. The sandwich-type theorems for these integral operators are also presented.
\end{abstract}

\section{Introduction}

Let $\mathcal{H}=\mathcal{H}(\mathbb{U})$ denote the class of analytic functions in the open unit disk $\mathbb{U}=\{z \in \mathbb{C}:|z|<1\}$. For $a \in \mathbb{C}$ and $n \in \mathbb{N}=\{1,2, \cdots\}$, let

$$
\mathcal{H}[a, n]=\left\{f \in \mathcal{H}: f(z)=a+a_{n} z^{n}+a_{n+1} z^{n+1}+\cdots\right\} .
$$

Let $f$ and $F$ be members of $\mathcal{H}$. The function $f$ is said to be subordinate to $F$, or $F$ is said to be superordinate to $f$, if there exists a function $w$ analytic in $\mathbb{U}$, with $w(0)=0$ and $|w(z)|<1$ for $z \in \mathbb{U}$, such that

$$
f(z)=F(w(z)) \quad(z \in \mathbb{U}) .
$$

In such a case, we write

$$
f \prec F \quad \text { or } \quad f(z) \prec F(z) .
$$

If the function $F$ is univalent in $\mathbb{U}$, then we have (cf. [12])

$$
f \prec F \quad \Longleftrightarrow f(0)=F(0) \text { and } \quad f(\mathbb{U}) \subset F(\mathbb{U}) \text {. }
$$

Definition 1.([12]) Let $\phi: \mathbb{C}^{2} \rightarrow \mathbb{C}$ and let $h$ be univalent in $\mathbb{U}$. If $p$ is analytic in $\mathbb{U}$ and satisfies the differential subordination:

$$
\phi\left(p(z), z p^{\prime}(z)\right) \prec h(z) \quad(z \in \mathbb{U}),
$$

Received January 24, 2012; Accepted February 23, 2012.

2000 Mathematics Subject Classification. Primary 30C80; Secondary 30C45.

Key words and phrases. Meromorphic functions, differential subordination, differential superordination, Sandwich-type theorems, integral operators.

This work was supported by the Pukyong National University Research Fund in 2011(PK2011-35) 
then $p$ is called a solution of the differential subordination. The univalent function $q$ is called a dominant of the solutions of the differential subordination, or more simply a dominant if $p \prec q$ for all $p$ satisfying (1.1). A dominant $\tilde{q}$ that satisfies $\tilde{q} \prec q$ for all subordinants $q$ of (1.1) is said to be the best dominant.

Definition 2.([13]) Let $\varphi: \mathbb{C}^{2} \rightarrow \mathbb{C}$ and let $h$ be analytic in $\mathbb{U}$. If $p$ and $\varphi\left(p(z), z p^{\prime}(z)\right)$ are univalent in $\mathbb{U}$ and satisfy the differential superordination:

$$
h(z) \prec \varphi\left(p(z), z p^{\prime}(z)\right) \quad(z \in \mathbb{U}),
$$

then $p$ is called a solution of the differential superordination. An analytic function $q$ is called a subordinant of the solutions of the differential superordination, or more simply a subordinant if $q \prec p$ for all $p$ satisfying (1.2). A univalent subordinant $\tilde{q}$ that satisfies $q \prec \tilde{q}$ for all subordinants $q$ of (1.2) is said to be the best subordinant.

Definition 3.([13]) We denote by $\mathcal{Q}$ the class of functions $f$ that are analytic and injective on $\overline{\mathbb{U}} \backslash E(f)$, where

$$
E(f)=\left\{\zeta \in \partial \mathbb{U}: \lim _{z \rightarrow \zeta} f(z)=\infty\right\},
$$

and are such that

$$
f^{\prime}(\zeta) \neq 0 \quad(\zeta \in \partial \mathbb{U} \backslash E(f)) .
$$

We also denote the class $A$ by

$$
A:=\left\{h \in \mathcal{H}[1,1]: h(z) h^{\prime}(z) \neq 0 \quad(z \in \mathbb{U} \backslash\{0\})\right\} .
$$

Let $\Sigma$ denote the class of functions of the form

$$
f(z)=\frac{1}{z}+\sum_{n=0}^{\infty} a_{n} z^{n}
$$

which are analytic in the punctured open unit disk $\mathbb{D}=\mathbb{U} \backslash\{0\}$. Let $\Sigma^{*}$ and $\Sigma_{k}$ be the subclasses of $\Sigma$ consisting of all functions which are, respectively, meromorphic starlike and meromorphic convex in $\mathbb{D}$ (see, for details, [5, 12]).

For a function $f \in \Sigma$, we introduce the following integral operators $I_{\beta, \gamma}$ defined by

$$
\begin{gathered}
I_{\beta, \gamma}(f)(z):=\left(\frac{\gamma-\beta}{z^{\gamma}} \int_{0}^{z} f^{\beta}(t) h^{\gamma-1}(t) h^{\prime}(t) d t\right)^{1 / \beta} \\
(f \in \Sigma ; \beta, \gamma \in \mathbb{C} ; \beta \in \mathbb{C} \backslash\{0\} ; \mathfrak{R}\{\gamma-\beta\}>0 ; h \in A) .
\end{gathered}
$$

Various developments associated with the integral operator $I_{\beta, \gamma}(f)$ defined by (1.3) have been extensively studied by many authors $[1,2,5-7]$ with suitable restrictions on the parameters $\beta$ and $\gamma$, and for $f$ belonging to some favoured classes of meromorphic functions. 
Moreover, making use of the principle of subordination between analytic functions, Miller et al. [14] and more recently, Owa and Srivastava [15] obtained some subordination preserving properties for certain integral operators. Moreover, Miller and Mocanu [13] considered differential superordinations, as the dual concept of differential subordinations (see also [3]). We also remark that some more interesting results related to subordination and subordination may be founded in [4]. In the present paper, we obtain the subordination and superordination preserving properties of the integral operator $I_{\beta, \gamma}$ defined by (1.3) with the sandwich-type theorem.

The following lemmas will be required in our present investigation.

Lemma 1.([10]) Suppose that the function $H: \mathbb{C}^{2} \rightarrow \mathbb{C}$ satisfies the following condition:

$$
\mathfrak{R}\{H(i s, t)\} \leq 0
$$

for all real $s$ and for all $t \leq-n\left(1+s^{2}\right) / 2(n \in \mathbb{N})$. If the function

$$
p(z)=1+p_{n} z^{n}+\cdots
$$

is analytic in $\mathbb{U}$ and

$$
\mathfrak{R}\left\{H\left(p(z), z p^{\prime}(z)\right)\right\}>0 \quad(z \in \mathbb{U}),
$$

then

$$
\mathfrak{R}\{p(z)\}>0 \quad(z \in \mathbb{U}) .
$$

Lemma 2.([11]) Let $\beta, \gamma \in \mathbb{C}$ with $\beta \neq 0$ and $h \in \mathcal{H}(\mathbb{U})$ with $h(0)=c$. If

$$
\mathfrak{R}\{\beta h(z)+\gamma\}>0 \quad(z \in \mathbb{U}),
$$

then the solution of the differential equation:

$$
q(z)+\frac{z q^{\prime}(z)}{\beta q(z)+\gamma}=h(z) \quad(z \in \mathbb{U} ; q(0)=c)
$$

is analytic in $\mathbb{U}$ and satisfies the following inequality given by

$$
\mathfrak{R}\{\beta q(z)+\gamma\}>0 \quad(z \in \mathbb{U}) .
$$

Lemma 3.([12]) Let $p \in \mathcal{Q}$ with $p(0)=a)$ and let

$$
q(z)=a+a_{n} z^{n}+\cdots \quad(q(z) \not \equiv a \quad \text { and } \quad n \in \mathbb{N})
$$

be analytic in $\mathbb{U}$. If $q$ is not subordinate to $p$, then there exist points $z_{0}=$ $r_{0} \mathrm{e}^{i \theta} \in \mathbb{U}$ and $\zeta_{0} \in \partial \mathbb{U} \backslash E(f)$, for which

$$
q\left(\mathbb{U}_{r_{0}}\right) \subset p(\mathbb{U}), q\left(z_{0}\right)=p\left(\zeta_{0}\right) \quad \text { and } \quad z_{0} q^{\prime}\left(z_{0}\right)=m \zeta_{0} p^{\prime}\left(\zeta_{0}\right) \quad(m \geq n) .
$$


Let

$$
N:=N(c)=\frac{|c| \sqrt{1+2 \mathfrak{R}\{c\}}+\mathfrak{I}\{c\}}{\mathfrak{R}\{c\}} \quad(c \in \mathbb{C} ; \mathfrak{R}\{c\}>0) .
$$

If $R$ is the univalent function defined in $\mathbb{U}$ by $R(z)=2 N z /\left(1-z^{2}\right)$, then the open door function (see, for details, [12]) defined by

$$
R_{c}(z):=R\left(\frac{z+b}{1+\bar{b} z}\right) \quad\left(z \in \mathbb{U} ; b=R^{-1}(c)\right) .
$$

Remark 1. The function $R_{c}$ defined by (1.4) is univalent in $\mathbb{U}, R_{c}(0)=c$ and $R_{c}(\mathbb{U})=R(\mathbb{U})$ is the complex plane with slits along the half-lines $\mathfrak{R}\{w\}=0$ and $\mathfrak{I}\{w\} \mid \geq N$.

Lemma 4. Let $\beta, \gamma \in \mathbb{C}$ with $\beta \neq 0$ and $\mathfrak{R}\{\gamma-\beta\}>0$ and let $h \in A$. If $f \in \Sigma_{\beta, \gamma}$, where

$$
\Sigma_{\beta, \gamma}:=\left\{f \in \Sigma: \beta \frac{z f^{\prime}(z)}{f(z)}+(\gamma-1) \frac{z h^{\prime}(z)}{h(z)}+1+\frac{z h^{\prime \prime}(z)}{h^{\prime}(z)} \prec R_{\gamma-\beta}(z)\right\}
$$

and $R_{\gamma-\beta}(z)$ is open door defined by (1.4) with $c=\gamma-\beta$, then

$$
I_{\beta, \gamma}(f) \in \Sigma, \quad z I_{\beta, \gamma}(f)(z) \neq 0 \quad(z \in \mathbb{U})
$$

and

$$
\mathfrak{R}\left\{\beta \frac{z\left(I_{\beta, \gamma}(f)(z)\right)^{\prime}}{I_{\beta, \gamma}(f)(z)}+\gamma\right\}>0 \quad(z \in \mathbb{U})
$$

where $I_{\beta, \gamma}$ is the integral operator defined by (1.3).

Remark 2 . The proof of Lemma 4 can be derived very easily by using the same techniques given in the proof of Miller and Mocanu [12].

A function $L(z, t)$ defined on $\mathbb{U} \times[0, \infty)$ is the subordination chain (or Loewner chain) if $L(\cdot, t)$ is analytic and univalent in $\mathbb{U}$ for all $t \in[0, \infty), L(z, \cdot)$ is continuously differentiable on $[0, \infty)$ for all $z \in \mathbb{U}$ and

$$
L(z, s) \prec L(z, t) \quad(z \in \mathbb{U} ; 0 \leq s<t) .
$$

Lemma 5.([13]) Let $q \in \mathcal{H}[a, 1]$ and $\mu: \mathbb{C}^{2} \rightarrow \mathbb{C}$. Also set

$$
\mu\left(q(z), z q^{\prime}(z)\right) \equiv h(z) \quad(z \in \mathbb{U}) .
$$

If

$$
L(z, t)=\mu\left(q(z), t z q^{\prime}(z)\right)
$$

is a subordination chain and $p \in \mathcal{H}[a, 1] \cap \mathcal{Q}$, then

$$
h(z) \prec \mu\left(p(z), z p^{\prime}(z)\right) \quad(z \in \mathbb{U})
$$

implies that

$$
q(z) \prec p(z) \quad(z \in \mathbb{U})
$$


Furthermore, if

$$
\mu\left(q(z), z q^{\prime}(z)\right)=h(z)
$$

has a univalent solution $q \in \mathcal{Q}$, then $q$ is the best subordinant.

Lemma 6.([16]) The function

$$
L(z, t)=a_{1}(t) z+\cdots
$$

with $a_{1}(t) \neq 0$ and $\lim _{t \rightarrow \infty}\left|a_{1}(t)\right|=\infty$. Suppose that $L(\cdot ; t)$ is analytic in $\mathbb{U}$ for all $t \geq 0, L(z ; \cdot)$ is continuously differentiable on $[0, \infty)$ for all $z \in \mathbb{U}$. If $L(z ; t)$ satisfies

$$
\mathfrak{R}\left\{\frac{\frac{z \partial L(z, t)}{\partial z}}{\frac{\partial L(z, t)}{\partial t}}\right\}>0 \quad(z \in \mathbb{U} ; 0 \leq t<\infty)
$$

and

$$
\left.|L(z ; t)| \leq K_{0}\left|a_{1}(t)\right| \quad\left(|z|<r_{0}<1 ; 0 \leq t<\infty\right)\right)
$$

for some positive constants $K_{0}$ and $r_{0}$, then $L(z ; t)$ is a subordination chain.

\section{Main results}

We begin by proving subordination theorem involving the integral operator $I_{\beta, \gamma}$ defined by (1.3) is contained in Theorem 1 below.

Theorem 1. Let $f, g \in \Sigma_{\beta, \gamma}$. Suppose that

$$
\operatorname{Re}\left\{1+\frac{z v^{\prime \prime}(z)}{v^{\prime}(z)}\right\}>-\rho \quad\left(z \in \mathbb{U} ; v(z):=z[z g(z)]^{\beta}\left[\frac{h(z)}{z}\right]^{\gamma-1} h^{\prime}(z)\right),
$$

where

$$
\rho=\frac{1+|\gamma-\beta-1|^{2}-\left|1-(\gamma-\beta-1)^{2}\right|}{4 \operatorname{Re}\{\gamma-\beta-1\}} \quad(\mathfrak{R}\{\gamma-\beta-1\}>0) .
$$

Then the subordination relation:

$$
z[z f(z)]^{\beta}\left[\frac{h(z)}{z}\right]^{\gamma-1} h^{\prime}(z) \prec z[z g(z)]^{\beta}\left[\frac{h(z)}{z}\right]^{\gamma-1} h^{\prime}(z) \quad(z \in \mathbb{U})
$$

implies that

$$
z\left[z I_{\beta, \gamma}(f)(z)\right]^{\beta} \prec z\left[z I_{\beta, \gamma}(g)(z)\right]^{\beta} \quad(z \in \mathbb{U}),
$$

where $I_{\beta, \gamma}$ is the integral operator defined by (1.3). Moreover, the function

$$
z\left[z I_{\beta, \gamma}(g)(z)\right]^{\beta}
$$

is the best dominant.

Proof. Let us define the functions $U$ and $V$ by

$$
U(z):=z\left[z I_{\beta, \gamma}(f)(z)\right]^{\beta} \text { and } V(z):=z\left[z I_{\beta, \gamma}(g)(z)\right]^{\beta},
$$


respectively. We note that $U$ and $V$ are well-defined by Lemma 4 . Without loss of generality, we can assume that $V$ is analytic and univalent on $\overline{\mathbb{U}}$ and that

$$
U^{\prime}(\zeta) \neq 0 \quad(|\zeta|=1) .
$$

We first show that, if the function $q$ is defined by

$$
q(z):=1+\frac{z V^{\prime \prime}(z)}{V^{\prime}(z)} \quad(z \in \mathbb{U})
$$

then

$$
\mathfrak{R}\{q(z)\}>0 \quad(z \in \mathbb{U}) .
$$

In terms of the function $v$ involved in (2.1), the definition (1.3) readily yields

$$
\left[z I_{\beta, \gamma}(g)(z)\right]^{\beta}\left[\beta \frac{z\left(I_{\beta, \gamma}(g)(z)\right)^{\prime}}{I_{\beta, \gamma}(g)(z)}+\gamma\right]=(\gamma-\beta)[z g(z)]^{\beta}\left(\frac{h(z)}{z}\right)^{\gamma-1} h^{\prime}(z) .
$$

We also have

$$
\beta \frac{z\left(I_{\beta, \gamma}(g)(z)\right)^{\prime}}{I_{\beta, \gamma}(g)(z)}=\frac{z V^{\prime}(z)}{V(z)}-\beta-1 .
$$

It follows from (2.7) and (2.8), we obtain

$$
(\gamma-\beta) v(z)=(\gamma-\beta-1) V(z)+z V^{\prime}(z)
$$

By a simple calculation with (2.9), we obtain the relationship:

$$
1+\frac{z v^{\prime \prime}(z)}{v^{\prime}(z)}=q(z)+\frac{z q^{\prime}(z)}{q(z)+\gamma-\beta-1} \equiv h(z) .
$$

From (2.1), we see that

$$
\mathfrak{R}\{h(z)+\gamma-\beta-1\}>0 \quad(z \in \mathbb{U})
$$

and by using Lemma 2, we conclude that the differential equation (2.10) has a solution $q \in \mathcal{H}(\mathbb{U})$ with

$$
q(0)=h(0)=1
$$

Let us put

$$
H(u, v)=u+\frac{v}{u+\gamma-\beta-1}+\rho,
$$

where $\rho$ is given by (2.2). From $(2.1),(2.10)$ and (2.11), we obtain

$$
\mathfrak{R}\left\{H\left(q(z), z q^{\prime}(z)\right)\right\}>0 \quad(z \in \mathbb{U}) .
$$

Now we proceed to show that

$$
\mathfrak{R}\{H(i s, t)\} \leq 0 \quad\left(s \in \mathbb{R} ; t \leq-\frac{\left(1+s^{2}\right)}{2}\right) .
$$


From (2.11), we have

$$
\begin{aligned}
\mathfrak{R}\{H(i s, t)\} & =\mathfrak{R}\left\{i s+\frac{t}{i s+\gamma-\beta-1}+\rho\right\} \\
& =\frac{t \mathfrak{R}\{\gamma-\beta-1\}}{|\gamma-\beta-1+i s|^{2}}+\rho . \\
& \leq-\frac{E_{\rho}(s)}{2|\gamma-\beta-1+i s|^{2}},
\end{aligned}
$$

where

$$
\begin{aligned}
E_{\rho}(s):= & (\mathfrak{R}\{\gamma-\beta-1\}-2 \rho) s^{2}-4 \rho(\mathfrak{R}\{\gamma-\beta-1\} s \\
& -2 \rho|\gamma-\beta-1|^{2}+\mathfrak{R}\{\gamma-\beta-1\} .
\end{aligned}
$$

We note that the coefficient of $s^{2}$ in the quadratic expression $E_{\rho}(s)$ given by (2.14) is positive or equal to zero and the quadratic expression by $s$ in (2.14) is a perfect square for the assumed value of $\rho$ given by (2.2). Hence from (2.13), we see that (2.12) holds true. Thus, by using Lemma 1, we conclude that

$$
\mathfrak{R}\{q(z)\}>0 \quad(z \in \mathbb{U}) .
$$

Hence the function $V$ defined by (2.5) is convex in $\mathbb{U}$.

Next, we prove that the subordination condition (2.3) implies that

$$
U(z) \prec V(z) \quad(z \in \mathbb{U})
$$

for the functions $U$ and $V$ defined by (2.5). For this purpose, we consider the function $L(z, t)$ given by

$$
L(z, t):=\frac{\gamma-\beta-1}{\gamma-\beta} V(z)+\frac{1+t}{\gamma-\beta} z V^{\prime}(z) \quad(z \in \mathbb{U} ; 0 \leq t<\infty) .
$$

Since $V$ is convex in $U$ and $\mathfrak{R}\{\gamma-\beta-1\}>0$, we obtain that

$$
\left.\frac{\partial L(z, t)}{\partial z}\right|_{z=z_{0}}=V^{\prime}(0)\left(1+\frac{t}{\gamma-\beta}\right) \neq 0 \quad(z \in \mathbb{U} ; 0 \leq t<\infty)
$$

and

$$
\mathfrak{R}\left\{\frac{\frac{z \partial L(z, t)}{\partial z}}{\frac{\partial L(z, t)}{\partial t}}\right\}=\mathfrak{R}\left\{\gamma-\beta-1+(1+t)\left(1+\frac{z V^{\prime \prime}(z)}{V^{\prime}(z)}\right)\right\}>0 \quad(z \in \mathbb{U}) .
$$

Furthermore, since $V$ is convex in $\mathbb{U}$, by using the well-known growth and distortion sharp inequalities (see [8]) for convex functions, we can prove that the second condition of Lemma 6 is satisfied. Therefore, by virtue of Lemma 6 , $L(z, t)$ is a subordination chain. We observe from the definition of a subordination chain that

$$
v(z)=\frac{\gamma-\beta-1}{\gamma-\beta} V(z)+\frac{1}{\gamma-\beta} z V^{\prime}(z)=L(z, 0)
$$

and

$$
L(z, 0) \prec L(z, t) \quad(z \in \mathbb{U} ; 0 \leq t<\infty) .
$$


This implies that

$$
L(\zeta, t) \notin L(\mathbb{U}, 0)=v(\mathbb{U}) \quad(\zeta \in \partial \mathbb{U} ; 0 \leq t<\infty) .
$$

Now suppose that $U$ is not subordinate to $V$, then by Lemma 3 , there exists points $z_{0} \in \mathbb{U}$ and $\zeta_{0} \in \partial \mathbb{U}$ such that

$$
U\left(z_{0}\right)=V\left(\zeta_{0}\right) \text { and } z_{0} U\left(z_{0}\right)=(1+t) \zeta_{0} V^{\prime}\left(\zeta_{0}\right) \quad(0 \leq t<\infty) .
$$

Hence we have

$$
\begin{aligned}
L\left(\zeta_{0}, t\right) & =\frac{\gamma-\beta-1}{\gamma-\beta} V\left(\zeta_{0}\right)+\frac{1+t}{\gamma-\beta} \zeta_{0} V^{\prime}\left(\zeta_{0}\right) \\
& =\frac{\gamma-\beta-1}{\gamma-\beta} U\left(z_{0}\right)+\frac{1}{\gamma-\beta} z_{0} U^{\prime}\left(z_{0}\right) \\
& =z_{0}\left[z_{0} f\left(z_{0}\right)\right]^{\beta}\left[\frac{h\left(z_{0}\right)}{z_{0}}\right]^{\gamma-1} h^{\prime}\left(z_{0}\right) \in v(\mathbb{U}),
\end{aligned}
$$

by virtue of the subordination condition (2.3). This contradicts the above observation that

$$
L\left(\zeta_{0}, t\right) \notin v(\mathbb{U}) .
$$

Therefore, the subordination condition (2.3) must imply the subordination given by (2.15). Considering $U=V$, we see that the function $G(z)$ is the best dominant. This evidently completes the proof of Theorem 1.

Remark 3 . We note that $\rho$ given by (2.2) in Theorem 1 satisfies the inequality:

$$
0<\rho \leq 1 / 2
$$

We next prove a solution to a dual problem of Theorem 1, in the sense that the subordinations are replaced by superordinations.

Theorem 2. Let $f, g \in \Sigma_{\beta, \gamma}$. Suppose that

$$
\mathfrak{R}\left\{1+\frac{z v^{\prime \prime}(z)}{v^{\prime}(z)}\right\}>-\rho \quad\left(z \in \mathbb{U} ; v(z):=z[z g(z)]^{\beta}\left[\frac{h(z)}{z}\right]^{\gamma-1} h^{\prime}(z)\right),
$$

where $\rho$ is given by (2.2), and $z[z f(z)]^{\beta}(h(z) / z)^{\gamma-1} h^{\prime}(z)$ is univalent in $\mathbb{U}$ and

$$
z\left[z I_{\beta, \gamma}(f)(z)\right]^{\beta} \in \mathcal{Q},
$$

where $I_{\beta, \gamma}$ is the integral operator defined by (1.3). Then the superordination relation:

$$
z[z g(z)]^{\beta}\left[\frac{h(z)}{z}\right]^{\gamma-1} h^{\prime}(z) \prec z[z f(z)]^{\beta}\left[\frac{h(z)}{z}\right]^{\gamma-1} h^{\prime}(z) \quad(z \in \mathbb{U})
$$

implies that

$$
z\left[z I_{\beta, \gamma}(g)(z)\right]^{\beta} \prec z\left[z I_{\beta, \gamma}(f)(z)\right]^{\beta} \quad(z \in \mathbb{U}) .
$$


Moreover, the function

$$
z\left[z I_{\beta, \gamma}(g)(z)\right]^{\beta}
$$

is the best subordinant.

Proof. Let us define the functions $U$ and $V$, respectively, by (2.5). We first note that by using (2.7) and (2.8), we obtain

$$
\begin{aligned}
v(z) & =\frac{\gamma-\beta-1}{\gamma-\beta} V(z)+\frac{1}{\gamma-\beta} z V^{\prime}(z) \\
& =: \mu\left(V^{\prime}(z), z V^{\prime}(z)\right) .
\end{aligned}
$$

After a simple calculation, the equation (2.17) yields the relationship:

$$
1+\frac{z v^{\prime \prime}(z)}{v^{\prime}(z)}=q(z)+\frac{z q^{\prime}(z)}{q(z)+\gamma-\beta-1},
$$

where function $q$ is defined by (2.6). Then by using the same method as in the proof of Theorem 1, we can prove that

$$
\mathfrak{R}\{q(z)\}>0 \quad(z \in \mathbb{U}),
$$

that is, that $V$ defined by (2.5) is convex(univalent) in $\mathbb{U}$.

Next, we prove that the superordination condition (2.16) implies that

$$
V(z) \prec U(z) \quad(z \in \mathbb{U}) .
$$

Now consider the function $L(z, t)$ defined by

$$
L(z, t):=\frac{\gamma-\beta-1}{\gamma-\beta} V(z)+\frac{t}{\gamma-\beta} z V^{\prime}(z) \quad(z \in \mathbb{U} ; 0 \leq t<\infty) .
$$

Since $V$ is convex in $\mathbb{U}$ and $\mathfrak{R}\{\gamma-\beta-1\}>0$, we can prove easily that $L(z, t)$ is a subordination chain as in the proof of Theorem 1 . Therefore according to Lemma 5, we conclude that the superordination condition (2.16) must imply the superordination given by (2.18). Furthermore, since the differential equation (2.17) has the univalent solution $G$, it is the best subordinant of the given differential superordination. Therefore we complete the proof of Theorem 2 .

If we combine Theorem 1 and Theorem 2, then we obtain the following sandwich-type theorem.

Theorem 3. Let $f, g_{k} \in \Sigma_{\beta, \gamma}(k=1,2)$. Suppose that

$\mathfrak{R}\left\{1+\frac{z v_{k}^{\prime \prime}(z)}{v_{k}^{\prime}(z)}\right\}>-\rho \quad\left(z \in \mathbb{U} ; v_{k}(z):=z\left[z g_{k}(z)\right]^{\beta}\left[\frac{h(z)}{z}\right]^{\gamma-1} h^{\prime}(z) ; k=1,2\right)$,

where $\rho$ is given by (2.2), and the function $z[z f(z)]^{\beta}[h(z) / z]^{\gamma-1} h^{\prime}(z)$ is univalent in $\mathbb{U}$ and

$$
z\left[z I_{\beta, \gamma}(f)(z)\right]^{\beta} \in \mathcal{Q},
$$


where $I_{\beta, \gamma}$ is the integral operator defined by (1.3). Then the subordination relation:

$$
v_{1}(z) \prec z[z f(z)]^{\beta}\left[\frac{h(z)}{z}\right]^{\gamma-1} h^{\prime}(z) \prec v_{2}(z) \quad(z \in \mathbb{U})
$$

implies that

$$
z\left[z I_{\beta, \gamma}\left(g_{1}\right)(z)\right]^{\beta} \prec z\left[z I_{\beta, \gamma}(f)(z)\right]^{\beta} \prec z\left[z I_{\beta, \gamma}\left(g_{2}\right)(z)\right]^{\beta} \quad(z \in \mathbb{U}) .
$$

Moreover, the functions

$$
z\left[z I_{\beta, \gamma}\left(g_{1}\right)(z)\right]^{\beta} \quad \text { and } \quad z\left[z I_{\beta, \gamma}\left(g_{2}\right)(z)\right]^{\beta}
$$

are the best subordinant and the best dominant, respectively.

The assumption of Theorem 3 , that the functions

$$
z[z f(z)]^{\beta}\left[\frac{h(z)}{z}\right]^{\gamma-1} h^{\prime}(z) \text { and } z\left[z I_{\beta, \gamma}(f)(z)\right]^{\beta}
$$

need to be univalent in $\mathbb{U}$, may be replaced by another conditions in the following result.

Corollary 1. Let $f, g_{k} \in \Sigma_{\beta, \gamma}(k=1,2)$. Suppose that the condition (2.19) is satisfied and

$\mathfrak{R}\left\{1+\frac{z \psi^{\prime \prime}(z)}{\psi^{\prime}(z)}\right\}>-\rho\left(z \in \mathbb{U} ; \psi(z):=z[z f(z)]^{\beta}\left[\frac{h(z)}{z}\right]^{\gamma-1} h^{\prime}(z) ; z f(z) \in \mathcal{Q}\right)$,

where $\rho$ is given by (2.2). Then the subordination relation:

$$
v_{1}(z) \prec z[z f(z)]^{\beta}\left[\frac{h(z)}{z}\right]^{\gamma-1} h^{\prime}(z) \prec v_{2}(z) \quad(z \in \mathbb{U})
$$

implies that

$$
z\left[z I_{\beta, \gamma}\left(g_{1}\right)(z)\right]^{\beta} \prec z\left[z I_{\beta, \gamma}(f)(z)\right]^{\beta} \prec z\left[z I_{\beta, \gamma}\left(g_{2}\right)(z)\right]^{\beta} \quad(z \in \mathbb{U}),
$$

where $I_{\beta, \gamma}$ is the integral operator defined by (1.3). Moreover, the functions

$$
z\left[z I_{\beta, \gamma}\left(g_{1}\right)(z)\right]^{\beta} \quad \text { and } \quad z\left[z I_{\beta, \gamma}\left(g_{2}\right)(z)\right]^{\beta}
$$

are the best subordinant and the best dominant, respectively.

Proof. In order to prove Corollary 1, we have to show that the condition $(2.20)$ implies the univalence of $\psi(z)$ and

$$
U(z):=z\left(z I_{\beta, \gamma}(f)(z)\right)^{\beta} .
$$

Since $0<\rho \leq 1 / 2$ from Remark 1 , the condition (2.20) means that $\psi$ is a closeto-convex function in $\mathbb{U}$ (see [9]) and hence $\psi$ is univalent in $\mathbb{U}$. Furthermore, by using the same techniques as in the proof of Theorem 3, we can prove the convexity (univalence) of $\mathbb{U}$ and so the details may be omitted. Therefore, by applying Theorem 3, we obtain Corollary 1. 
By setting $\gamma-\beta=3$ in Theorem 3 , so that $\rho=1 / 4$, we deduce the following consequence of Theorem 3 .

Corollary 2. Let $f, g_{k} \in \Sigma_{\beta, \beta+3}(k=1,2)$. Suppose that

$\mathfrak{R}\left\{1+\frac{z v_{k}^{\prime \prime}(z)}{v_{k}^{\prime}(z)}\right\}>-\frac{1}{4}\left(z \in \mathbb{U} ; v_{k}(z):=z\left[z g_{k}(z)\right]^{\beta}\left[\frac{h(z)}{z}\right]^{\gamma-1} h^{\prime}(z) ; k=1,2\right)$,

and the function $z[z f(z)]^{\beta}[h(z) / z]^{\gamma-1} h^{\prime}(z)$ is univalent in $\mathbb{U}$ and

$$
z\left[z I_{\beta, \beta+3}(f)(z)\right]^{\beta} \in \mathcal{Q},
$$

where $I_{\beta, \beta+3}$ is the integral operator defined by (1.3) with $\gamma=\beta+3$. Then the subordination relation:

$$
v_{1}(z) \prec z[z f(z)]^{\beta}\left[\frac{h(z)}{z}\right]^{\gamma-1} h^{\prime}(z) \prec v_{2}(z) \quad(z \in \mathbb{U})
$$

implies that

$$
z\left[z I_{\beta, \beta+3}\left(g_{1}\right)(z)\right]^{\beta} \prec z\left[z I_{\beta, \beta+3}(f)(z)\right]^{\beta} \prec z\left[z I_{\beta, \beta+3}\left(g_{2}\right)(z)\right]^{\beta} \quad(z \in \mathbb{U}) .
$$

Moreover, the functions

$$
z\left[z I_{\beta, \beta+3}\left(g_{1}\right)(z)\right]^{\beta} \quad \text { and } \quad z\left[z I_{\beta, \beta+3}\left(g_{2}\right)(z)\right]^{\beta}
$$

are the best subordinant and the best dominant, respectively.

If we take $\gamma-\beta=2+i$ in Theorem 3 , then we easily to led to the following result.

Corollary 3. Let $f, g_{k} \in \Sigma_{\beta, \beta+2+i}(k=1,2)$. Suppose that

$\mathfrak{R}\left\{1+\frac{z v_{k}^{\prime \prime}(z)}{v_{k}^{\prime}(z)}\right\}>-\frac{3-\sqrt{5}}{4}\left(z \in \mathbb{U} ; v_{k}(z):=z\left[z g_{k}(z)\right]^{\beta}\left[\frac{h(z)}{z}\right]^{\gamma-1} h^{\prime}(z) ; k=1,2\right)$,

and the function $z[z f(z)]^{\beta}[h(z) / z]^{\gamma-1} h^{\prime}(z)$ is univalent in $\mathbb{U}$ and

$$
z\left[z I_{\beta, \beta+2+i}(f)(z)\right]^{\beta} \in \mathcal{Q},
$$

where $I_{\beta, \beta+2+i}$ is the integral operator defined by (1.3) with $\gamma=\beta+2+i$. Then the subordination relation:

$$
v_{1}(z) \prec z[z f(z)]^{\beta}\left[\frac{h(z)}{z}\right]^{\gamma-1} h^{\prime}(z) \prec v_{2}(z) \quad(z \in \mathbb{U})
$$

implies that

$z\left[z I_{\beta, \beta+2+i}\left(g_{1}\right)(z)\right]^{\beta} \prec z\left[z I_{\beta, \beta+2+i}(f)(z)\right]^{\beta} \prec z\left[z I_{\beta, \beta+2+i}\left(g_{2}\right)(z)\right]^{\beta} \quad(z \in \mathbb{U})$.

Moreover, the functions

$$
z\left[z I_{\beta, \beta+2+i}\left(g_{1}\right)(z)\right]^{\beta} \quad \text { and } \quad z\left[z I_{\beta, \beta+2+i}\left(g_{2}\right)(z)\right]^{\beta}
$$

are the best subordinant and the best dominant, respectively. 


\section{References}

[1] S. K. Bajpai, A note on a class of meromorphic univalent functions, Rev. Roumaine Math. Pures Appl. 22 (1977), 295-297.

[2] S. S. Bhoosnurmath and S. R. Swamy, Certain integrals for classes of univalent meromorphic functions, Ganita 44 (1993), 19-25.

[3] T. Bulboacă, A class of superordination-preserving integral operators, Indag. Math. N. S. 13 (2002), 301-311.

[4] N. E. Cho and H. M. Srivastava, A class of nonlinear integral operators preserving subordination and superordination, Integral Transforms Spec. Funct. 18 (2007), 95-107.

[5] A. Dernek, Certain classes of meromorphic functions, Ann. Univ. Mariae CurieSkłodowska Sect. A 42 (1988), 1-8.

[6] S. P. Dwivedi, G. P. Bhargava and S. K. Shukla, On some classes of meromorphic univalent functions, Rev. Roumaine Math. Pures Appl. 25 (1980), 209-215.

[7] R. M. Goel and N. S. Sohi, On a class of meromorphic functions, Glas. Mat. 17(37) (1981), 19-28.

[8] D. J. Hallenbeck and T. H. MacGregor, Linear problems and convexity techniques in geometric function theory, Pitman Publishing Limited, London, 1984.

[9] W. Kaplan, Close-to-convex schlicht functions, Michigan Math. J. 2 (1952), 169-185.

[10] S. S. Miller and P. T. Mocanu, Differential subordinations and univalent functions, Michigan Math. J. 28 (1981), 157-171.

[11] _ Univalent solutions of Briot-Bouquet differential equations, J. Different. Equations 56 (1985), 297-309.

[12] _ Differential Subordinations, Theory and Applications, Marcel Dekker, Inc., New York, Basel, 2000

[13] _ Subordinants of differential superordinations, Complex Var. Theory Appl. 48 (2003), 815-826.

[14] S. S. Miller, P. T. Mocanu and M. O. Reade, Subordination-preserving integral operators, Trans. Amer. Math. Soc. 283 (1984), 605-615.

[15] S. Owa and H. M. Srivastava, Some subordination theorems involving a certain family of integral operators, Integral Transforms Spec. Funct. 15 (2004), 445-454.

[16] Ch. Pommerenke, Univalent Functions, Vanderhoeck and Ruprecht, Göttingen, 1975.

NAK EUN CHO

Department of Applied Mathematics, Pukyong National University, Pusan 608737, KOREA

E-mail address: necho@pknu.ac.kr 